\title{
Inorganic Phosphors for Temperature Sensing in Hydrogen-Rich Environment
}

\author{
Benson Shu Ip ${ }^{1}$, Yuriy Syvenkyy², Yurij Mozharivskyj $j^{1}$ \\ ${ }^{1}$ Dept. of Chemistry and Chemical Biology, McMaster University, Hamilton, ON, Canada L9H 6H1, \\ ${ }^{2}$ Photon Control Inc, Richmond, BC, Canada V6V 1 V2 \\ mozhar@mcmaster.ca
}

\begin{abstract}
:
Inorganic phosphor materials can be used for temperature measurements since the lifetime of phosphorescence is temperature dependent. For practical applications, the lifetime in the range of milliseconds to microseconds is required and this condition limits the choice of activators to $\mathrm{Cr}^{3+}$ and $\mathrm{Mn}^{4+}$ among the $3 d$ metals. The working environment and instrumental features puts additional constraints on the chemical and temperature stability, absorption and emission profiles of phosphors. This paper discusses development of phosphor materials for operation in the hydrogen-rich environments, such as Canadian oil fields. Presence of hydrocarbons leads to saturation of optic fibers with hydrogen and attenuation of light above $705 \mathrm{~nm}$, the emission wavelength of the $\mathrm{Cr}^{3+}$ doped $\mathrm{Y}_{3} \mathrm{Al}_{5} \mathrm{O}_{12}$ temperature phosphor. Using chemical tools, a phosphor with longer emission wavelength was developed. This phosphor also meets the other requirements imposed by the current instrumentation.
\end{abstract}

Key words: temperature sensors, inorganic phosphors, emission, lifetime.

\section{Background and requirements}

Intensity and decay time of the light emitted by a phosphor are temperature dependent and can be used for temperature measurements. In one approach, the intensity ratio for two emission lines is used; in the other, the decay rate of the intensity of one emission line is utilized. The latter method is technologically simpler and is incorporated into the fiber optic temperature sensors developed by Photon Control Inc. Intensity, $I$, of the emitted light decreases exponentially with time, $t, \quad I=I_{0} \exp (-t / \tau)$, with $I_{0}$ being the initial intensity and $\tau$ the decay time (or lifetime). Typically, the decay time is temperature dependent and decreases linearly (on the log scale) with temperature. In a fiberoptic sensor, a phosphor is excited with a laser pulse and the decay time is determined by monitoring the intensity of the emitted light. For phosphor materials, the decay time of $10^{-6}-10^{-3}$ $\mathrm{s}$ is preferred as it can be precisely and inexpensively measured.

The $\mathrm{Cr}^{3+}\left(3 d^{3}\right)$ cation is extensively used in the phosphor materials due to its high efficiency, long lifetime, suitable absorption and emission wavelengths. The Photon Control Inc. uses the Cr-doped $\mathrm{Y}_{3} \mathrm{Al}_{5} \mathrm{O}_{12}$ garnet for temperature detection in the non-hydrogen rich environments. However, this material cannot be employed when hydrogen is present.

For operation in the hydrogen-rich environment and to be compatible with the current instrumentation, a new phosphor should be (1) chemically inert, have (2) photoluminescent $(\mathrm{PL})$ emission in the $750-850 \mathrm{~nm}$ range, (3) excitation between 530 and $700 \mathrm{~nm}$, and (4) decay time of milliseconds at room temperature.
Approach
The molecular states for $\mathrm{Cr}^{3+}$ in an octahedral crystal field as well as the effect of the crystal field strength on their energy are presented in the Tagano-Sunabe diagram (Fig. 1). The PL emission in $\mathrm{Cr}^{3+}$ originates from the transition from the ${ }^{2} \mathrm{E}$ state to the ${ }^{4} \mathrm{~A}_{2}$ one. To shift the emission to a longer wavelength (lower energy) one must decrease the energy separation between these two states. As the slope of the ${ }^{2} E$ curve is small, the effect of the crystal field on the emission energy will also be small. To tune the $\mathrm{PL}$ wavelength, a significant weakening of the crystal field is required. This is 
achieved by introducing large atoms into the host structure.

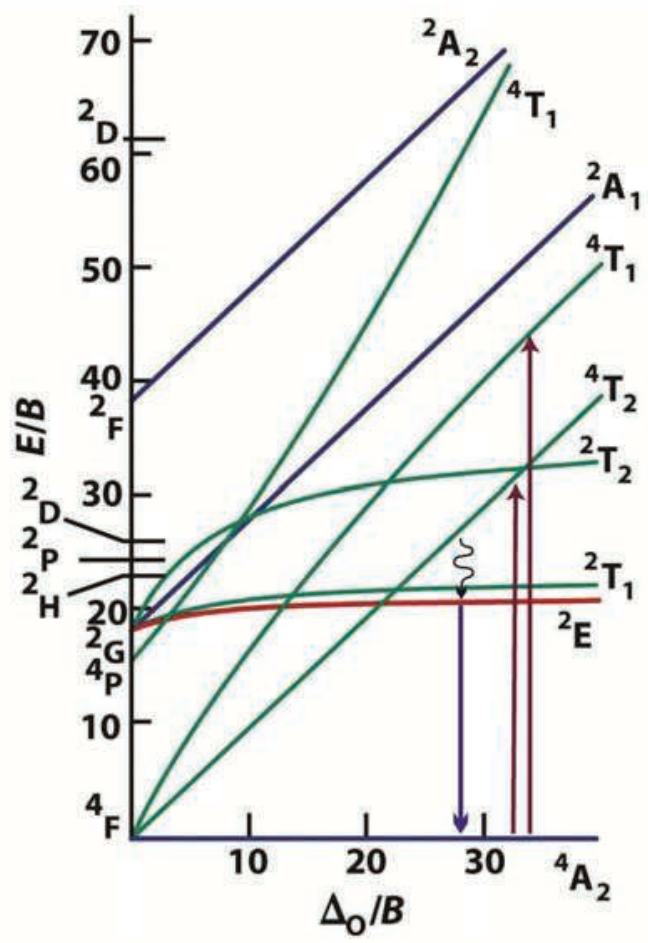

Fig. 1. Molecular states for $\mathrm{Cr}^{3+}$ in an octahedral field. The ${ }^{2} E \rightarrow{ }^{4} A_{2}$ transition yields PL.

\section{Results}

The reduction in the crystal field and shift of the emission wavelength was achieved by introducing larger atoms into the host structure of $\mathrm{Y}_{3} \mathrm{Al}_{5} \mathrm{O}_{12}$. Fig. 2 shows the absorption and emission profiles of the $\mathrm{Cr}^{3+}$-doped $\mathrm{Y}_{3} \mathrm{Al}_{5} \mathrm{O}_{12}$ while Fig. 3 the same for the targeted phosphor material.

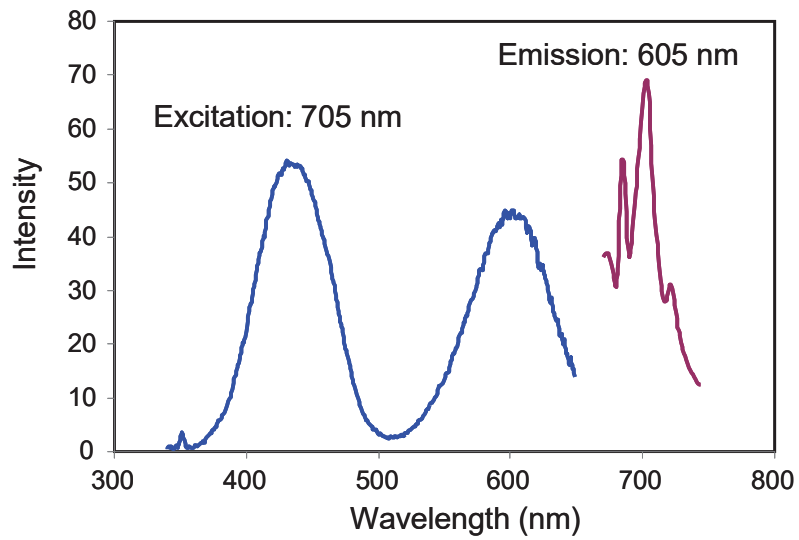

Fig. 2. Excitation and emission profiles of $\mathrm{Cr}^{3+}$ doped $\mathrm{Y}_{3} \mathrm{Al}_{5} \mathrm{O}_{12}$.

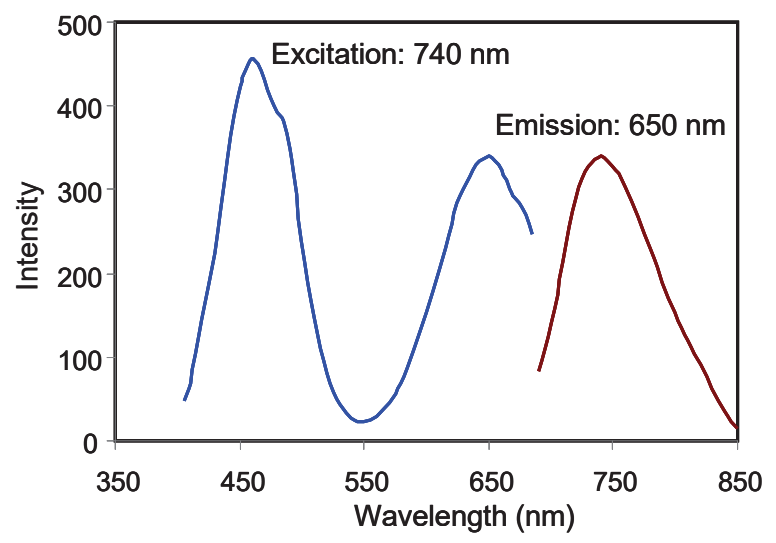

Fig. 3. Excitation and emission profiles of $\mathrm{Cr}^{3+}$ doped targeted phosphor.

The new phosphor has a lifetime of $0.103 \mathrm{~ms}$ at room temperature. While its lifetime and emission intensity decreased, they are still within required operational parameters. On the other hand, chemical stability and absorption fully meet the target values. 